\title{
ON UNSTABLE AND NEUTRAL SPECTRA OF INCOMPRESSIBLE INVISCID AND VISCID FLUIDS ON THE 2D TORUS
}

\author{
BY \\ VINCENT XIAOSONG LIU
}

Department of Mathematics, Stanford University, Stanford, California

\begin{abstract}
We completely determine all the unstable modes and neutral modes for the Kolmogorov flow $\left(\sin \left(2 x_{2}\right), 0\right)$ on the two-dimensional torus $[0,2 \pi] \times[0,2 \pi]$ for both the Navier-Stokes equations and the Euler equations. We also give comments on "vorticity generation".
\end{abstract}

1. Main Results. It is a difficult problem to determine every unstable and neutral direction for the linearized Navier-Stokes equations around a steady flow for any large Reynolds numbers, unless the linear operator is self-adjoint or its unstable and neutral spectra are trivial. In this paper, for the special basic flow $\left(\sin \left(2 x_{2}\right), 0\right)$ on the two-dimensional torus $[0,2 \pi] \times[0,2 \pi]$, we give all the unstable modes and neutral modes for the Navier-Stokes equations for any Reynolds numbers. We also determine all the unstable modes and neutral modes for the corresponding Euler equations (that is, the case when the Reynolds number is infinity). We show that the unstable eigenvalues for the linearized Navier-Stokes equations approach the unstable eigenvalues for the linearized Euler equations when the viscosity approaches zero. We believe this is the first nontrivial example in which the linearized operators are not self-adjoint but all the unstable and neutral modes can be determined theoretically. We also comment on Vishik and Friedlander's result [18] on hydrodynamic stability.

The result here is a starting point for our study on stationary and Hopf bifurcations of the Navier-Stokes equations on the 2D torus (cf. [9]), and also for our future theoretical investigations on more complex dynamical behavior of the Navier-Stokes equations on the $2 \mathrm{D}$ torus.

Let $\Omega=[0,2 \pi] \times[0,2 \pi]$ be the $2 \mathrm{D}$ torus, and let $H$ be the subspace of $\left(L^{2}(\Omega)\right)^{2}$ that consists of sinusoidal periodic flows on $\Omega$ with space average zero. An orthonor-

Received December 11, 1992.

1991 Mathematics Subject Classification. Primary 76D05, 76E05, 76N10, 35Q30.

Key words and phrases. Navier-Stokes equations, Euler equations, spectrum, stability.

(C)1995 Brown University 
mal basis of $H$ is given by

$$
\begin{aligned}
& W_{\left(k_{1}, k_{2}\right)}=\frac{1}{\sqrt{2} \pi \sqrt{k_{1}^{2}+k_{2}^{2}}}\left(\begin{array}{c}
k_{2} \\
-k_{1}
\end{array}\right) \cos \left(k_{1} x_{1}+k_{2} x_{2}\right), \\
& W_{\left(k_{1}, k_{2}\right)}^{\prime}=\frac{1}{\sqrt{2} \pi \sqrt{k_{1}^{2}+k_{2}^{2}}}\left(\begin{array}{c}
k_{2} \\
-k_{1}
\end{array}\right) \sin \left(k_{1} x_{1}+k_{2} x_{2}\right),
\end{aligned}
$$

where $\left(k_{1}, k_{2}\right) \in K$ and

$$
K=\left\{\left(k_{1}, k_{2}\right) \mid k_{1}>0 \text { or } k_{1}=0, k_{2}>0\right\} .
$$

Let $u_{0}=W_{(0,2)}^{\prime}=\left(\frac{1}{\sqrt{2} \pi} \sin \left(2 x_{2}\right), 0\right)$ be our basic steady flow. By linearizing the Navier-Stokes equations around $u_{0}$, we get the following linear operator in $H$,

$$
L_{\nu} w=-\nu A w-B\left(u_{0}, w\right)-B\left(w, u_{0}\right),
$$

where

$$
\begin{gathered}
A u=-P \Delta u=-\Delta u, \\
B(v, w)=P[(v \cdot \nabla) w],
\end{gathered}
$$

$P$ is the orthogonal projection onto $H$ in $\left(L^{2}(\Omega)\right)^{2}, \nu=1 / R>0$, and $R$ is the Reynolds number.

When $\nu=0$, we get the corresponding linearized operator for the Euler equations in $H$,

$$
L_{0} w=-B\left(u_{0}, w\right)-B\left(w, u_{0}\right) .
$$

Considering the eigenvalue problem for $L_{\nu}(\nu \geq 0)$ :

$$
L_{\nu} W=\eta W,
$$

if the real part $\operatorname{Re} \eta$ of $\eta$ is positive, the disturbance is unstable and the corresponding eigenvectors are called unstable modes. If $\operatorname{Re} \eta<0$, the disturbance is damped and the eigenvectors are called stable modes. If $\operatorname{Re} \eta=0$, there is sustained oscillation and the corresponding eigenvectors are called neutral modes [6]. It is well known that for the operator $L_{\nu}(\nu>0)$, there are only finitely many unstable and neutral modes (see, for example, [15]). This is not true for $L_{0}$; see Theorem 2 below for an example.

Now we give our main results.

THEOREM 1. For the operator $L_{\nu}(\nu>0)$, there exist positive constants $\nu_{0}, \nu_{1}$ $\left(\nu_{1}<\nu_{0}\right)$ such that
A. If $\nu>\nu_{0}$, then all the eigenvalues of $L_{\nu}$ have negative real parts.
B. If $\nu=\nu_{0}$, then $\eta=0$ is an eigenvalue and the corresponding eigenspace is two dimensional; all the other eigenvalues have negative real parts.
C. If $0<\nu<\nu_{0}$, then there is a unique positive eigenvalue $\eta(\nu)$ and the corresponding eigenspace is two dimensional; all the other eigenvalues have 
negative real parts (except those possibly unstable and neutral modes from D below). $\eta(\nu)$ satisfies

$$
\sqrt{\frac{89}{20 \cdot 34}} \frac{1}{\pi}-5 \nu<\eta(\nu)<\sqrt{\frac{3}{20}} \frac{1}{\pi}-\nu .
$$

D. If $0<\nu \leq \nu_{1}$, in addition to case $\mathrm{C}$ above, there also exists a conjugate pair of eigenvalues $\eta_{1}(\nu), \overline{\eta_{1}(\nu)}$ such that

$$
\begin{aligned}
0 & <\operatorname{Re} \eta_{1}(\nu)<\sqrt{\frac{3}{40}} \frac{1}{\pi}-2 \nu \quad \text { if } 0<\nu<\nu_{1}, \\
0 & =\operatorname{Re} \eta_{1}(\nu) \quad \text { if } \nu=\nu_{1}, \\
\frac{\sqrt{5}-\sqrt{3}}{2 \sqrt{10} \pi} & <\operatorname{Im} \eta_{1}(\nu)<\frac{\sqrt{5}+\sqrt{3}}{2 \sqrt{10} \pi} \quad \text { if } 0<\nu \leq \nu_{1} .
\end{aligned}
$$

The corresponding eigenspace is two dimensional and all the other eigenvalues have negative real parts (except the positive eigenvalue from $\mathrm{C}$ above).

Moreover, estimates for $\nu_{0}, \nu_{1}$ are

$$
\begin{aligned}
\sqrt{\frac{89}{34}} \frac{1}{10 \pi} & <\nu_{0}<\sqrt{3} \frac{1}{10 \pi}, \\
0 & <\nu_{1}<\sqrt{\frac{3}{2}} \frac{1}{20 \pi} .
\end{aligned}
$$

Remark. Part D of Theorem 1 will give a Hopf bifurcation for the Navier-Stokes equations; the details will be given in [9].

THEOREM 2. For the operator $L_{0}$

A. There is only one positive eigenvalue $\eta_{0}$ and the corresponding eigenspace is two dimensional; $\eta_{0}$ satisfies

$$
\sqrt{\frac{89}{20 \cdot 34}} \frac{1}{\pi}<\eta_{0}<\sqrt{\frac{3}{20}} \frac{1}{\pi}
$$

B. There is a unique conjugate pair of eigenvalues $\eta_{1}, \overline{\eta_{1}}$ with positive real part and the corresponding eigenspace is two dimensional; $\eta_{1}$ satisfies

$$
\begin{aligned}
& 0<\operatorname{Re} \eta_{1}<\sqrt{\frac{3}{40} \frac{1}{\pi}}, \\
& \frac{\sqrt{5}-\sqrt{3}}{2 \sqrt{10} \pi}<\operatorname{Im} \eta_{1}<\frac{\sqrt{5}+\sqrt{3}}{2 \sqrt{10} \pi} .
\end{aligned}
$$

C. Every imaginary number is an eigenvalue and the corresponding eigenspace is infinite dimensional.

D. All the other eigenvalues have negative real parts.

REMARK. The neutral modes in $\mathrm{C}$ of Theorem 2 only belong to the space $\left(L^{2}(\Omega)\right)^{2}$; they do not belong to the space $\left(H^{1}(\Omega)\right)^{2}$ (see Lemma 5 below) except for those trivial neutral modes corresponding to the eigenvalue 0 . 
THEOREM 3. For the eigenvalues with positive real parts in Theorem 1 and Theorem 2 ,

$$
\begin{aligned}
\lim _{\nu \rightarrow 0} \eta(\nu) & =\eta_{0}, \\
\lim _{\nu \rightarrow 0} \eta_{1}(\nu) & =\eta_{1} .
\end{aligned}
$$

REMARK. One can also completely determine all the unstable and neutral modes for these basic flows,

by using the same technique.

$$
W_{\left(k_{1}, k_{2}\right)} \text { and } W_{\left(k_{1}, k_{2}\right)}^{\prime},
$$

We will prove Theorem 1 and Theorem 2 by applying the technique of $[7,8]$. Since here we need to completely determine all the unstable and neutral modes, the situation is more complex. In Sec. 2, we reduce our eigenvalue problem to certain three-term recurrence relations. In Sec. 3, we recall some results of continued fractions and minimal solutions. Section 4 will prove Theorem 1 and Theorem 2. Section 5 gives some lemmas and proof of Theorem 3. In Sec. 6, we will give comments on a result of Vishik and Friedlander [18].

2. The reduction of the problem. We first recall the procedure of $[7,8]$ of transforming the eigenvalue problem (3) for the operator $L_{\nu}(\nu \geq 0)$ to some three-term recurrence relations among the Fourier coefficients of the eigenvector (see also [11, 19]). In the eigenvalue problem (3), since the even and odd part of $W$ give exactly the same equation, respectively, we only need to consider the even part; so we assume the eigenvector $W$ is an even function:

$$
W=\sum_{\left(k_{1}, k_{2}\right) \in K} a_{\left(k_{1}, k_{2}\right)} W_{\left(k_{1}, k_{2}\right)} .
$$

Put the above $W$ into (3) for each $k_{1} \geq 0$. We get the following recurrence relations for $a_{\left(k_{1}, k_{2}\right)}$ for each fixed $k_{1} \geq 0$ (see, $\left.[7,8]\right)$ :

$$
\begin{aligned}
& \left(\nu\left(k_{1}^{2}+k_{2}^{2}\right)+\eta\right) a_{\left(k_{1}, k_{2}\right)} \\
& \quad+a_{\left(k_{1}, k_{2}-2\right)} \frac{k_{1}\left(k_{1}^{2}+\left(k_{2}-2\right)^{2}-4\right)}{2 \sqrt{2} \pi \sqrt{k_{1}^{2}+\left(k_{2}-2\right)^{2}} \sqrt{k_{1}^{2}+k_{2}^{2}}} \\
& \quad-a_{\left(k_{1}, k_{2}+2\right)} \frac{k_{1}\left(k_{1}^{2}+\left(k_{2}+2\right)^{2}-4\right)}{2 \sqrt{2} \pi \sqrt{k_{1}^{2}+\left(k_{2}+2\right)^{2}} \sqrt{k_{1}^{2}+k_{2}^{2}}}=0 \quad \text { for }\left(k_{1}, k_{2}\right) \in K .
\end{aligned}
$$

For each $k_{1}>0$, we let

$$
\begin{aligned}
b_{\left(k_{1}, k_{2}\right)} & =\frac{k_{1}^{2}+k_{2}^{2}-4}{\sqrt{k_{1}^{2}+k_{2}^{2}}} a_{\left(k_{1}, k_{2}\right)}, \\
c_{\left(k_{1}, k_{2}\right)} & =\frac{2 \sqrt{2} \pi\left(k_{1}^{2}+k_{2}^{2}\right)\left(\nu\left(k_{1}^{2}+k_{2}^{2}\right)+\eta\right)}{k_{1}\left(k_{1}^{2}+k_{2}^{2}-4\right)} .
\end{aligned}
$$


Then from (16), we get for each fixed $k_{1}>0$,

$$
c_{\left(k_{1}, k_{2}\right)} b_{\left(k_{1}, k_{2}\right)}+b_{\left(k_{1}, k_{2}-2\right)}-b_{\left(k_{1}, k_{2}+2\right)}=0 \quad \forall k_{2},
$$

and if $k_{1}=0$, from (16), we get

$$
\left(\nu k_{2}^{2}+\eta\right) a_{\left(0, k_{2}\right)}=0 \text { for } \forall k_{2}>0 .
$$

So the eigenvalue problem of solving (3) becomes the problem of solving (19) or (20), respectively. Since $W$ is an eigenvector, we need to solve (19) and (20) such that $W \in H$.

3. Three-term recurrence relations and minimal solution. Consider the "half" threeterm recurrence relations

$$
a_{n} c_{n}+c_{n-1}-c_{n+1}=0, \quad n=1,2, \ldots,
$$

and the "full" three-term recurrence relations

$$
a_{n} c_{n}+c_{n-1}-c_{n+1}=0, \quad n=0, \pm 1, \pm 2, \ldots,
$$

where $a_{n}, c_{n}$ are complex numbers.

We call a nontrivial solution $\left\{c_{n}\right\}$ of $(21)$ a minimal solution (see, $\left.[4,5]\right)$ if there is another solution $\left\{d_{n}\right\}$ of $(21)$ such that

$$
\lim _{n \rightarrow \infty} \frac{c_{n}}{d_{n}}=0 .
$$

Similarly, we call a nontrivial solution $\left\{c_{n}\right\}$ of $(22)$ a minimal solution if there is another solution $\left\{d_{n}\right\}$ of (22) such that

$$
\lim _{|n| \rightarrow \infty} \frac{c_{n}}{d_{n}}=0 .
$$

Now we recall Theorem 5.7 of [5] about a minimal solution.

The difference equation (21) has a minimal solution $\left\{c_{n}\right\}$ if and only if the continued fraction

$$
\frac{-1}{a_{1}+\frac{1}{a_{2}+\cdots}} \text {. }
$$

converges (to a finite number or infinity). Furthermore, if (23) converges, then

$$
\frac{c_{k}}{c_{k-1}}=\frac{-1}{a_{k}+\frac{1}{a_{k+1}+\cdots}}, \quad \forall k=1,2, \ldots
$$

From this result, we get

Lemma 1. The difference equation (22) has a minimal solution $\left\{c_{n}\right\}$ if and only if

$$
a_{0}+\frac{1}{a_{-1}+\frac{1}{a_{-2}+\cdots}}=\frac{-1}{a_{1}+\frac{1}{a_{2}+\cdots}} .
$$

Moreover, $c_{1} / c_{0}$ equals this expression. 
Proof. We observe $\left\{c_{n}\right\}_{n=-\infty}^{+\infty}$ is a minimal solution of (22) if and only if $\left\{c_{n}\right\}_{n=0}^{+\infty}$ is a minimal solution of $(21)$ and $\left\{c_{n}\right\}_{n=-\infty}^{1}$ is a minimal solution of

$$
a_{n} c_{n}+c_{n-1}-c_{n+1}=0, \quad \forall n=0,-1,-2, \ldots
$$

From the above result, we have that $\left\{c_{n}\right\}_{n=1}^{+\infty}$ is a minimal solution of (21) if and only if

$$
\frac{c_{1}}{c_{0}}=\frac{-1}{a_{1}+\frac{1}{a_{2}+\cdots}} .
$$

We observe that $\left\{c_{n}\right\}_{n=-\infty}^{1}$ is a minimal solution of (26) if and only if $\left\{c_{n}^{\prime}=\right.$ $\left.(-1)^{n} c_{-n}\right\}_{n=-1}^{\infty}$ is a minimal solution of

$$
a_{-n} c_{n}^{\prime}+c_{n-1}^{\prime}-c_{n+1}^{\prime}=0, \quad \forall n=0,1, \ldots,
$$

so by the above result, if and only if

$$
\frac{c_{0}^{\prime}}{c_{-1}^{\prime}}=\frac{c_{0}}{-c_{1}}=\frac{-1}{a_{0}+\frac{1}{a_{-1}+\cdots}} .
$$

Thus from (27) and (28), we have proved Lemma 1.

Now for the difference equation (22), if we assume

$$
\operatorname{Re} a_{n}>0, \quad \forall n \neq 0,1,
$$

and

$$
\prod_{k=2}^{k=\infty} \frac{1}{\operatorname{Re} a_{k}}=0 \quad \text { and } \quad \prod_{k=-\infty}^{k=-1} \frac{1}{\operatorname{Re} a_{k}}=0
$$

then we have

Lemma 2. There is a nontrivial solution $\left\{c_{n}\right\}$ of (22) such that

$$
\lim _{|n| \rightarrow \infty} c_{n}=0
$$

if and only if the following equation is true:

$$
a_{0}+\frac{1}{a_{-1}+\frac{1}{a_{-2}+\cdots}}=\frac{-1}{a_{1}+\frac{1}{a_{2}+\cdots}} .
$$

Moreover, this nontrivial solution is unique within a constant factor and satisfies

$$
\begin{aligned}
c_{n} & \neq 0, \quad \forall n, \\
\operatorname{Re} \frac{c_{n}}{c_{n-1}}<0, \quad \forall n \geq 2 & \text { and } \operatorname{Re} \frac{c_{n}}{c_{n-1}}>0, \quad \forall n \leq 0 .
\end{aligned}
$$

Proof. See the proof of Theorem 2 of [7] and observe the following.

Let

$$
t_{k}(z)=\frac{1}{a_{k}+z} \text { and } T_{n}(z)=t_{1} \circ t_{2} \circ \cdots \circ t_{n}(z),
$$

where $\operatorname{Re} a_{k}>0, k=1,2, \ldots, n$. 
Let $U=\{z \in \mathbb{C} \mid \operatorname{Re} z \geq 0\}$. Then

$$
T_{n}(U) \text { is a closed disk with radius less or equal to } \frac{1}{2} \prod_{j=1}^{n-1} \frac{1}{\left(\operatorname{Re} a_{j}\right)^{2}} \frac{1}{\operatorname{Re} a_{n}},
$$

and

$$
T_{n}(U) \subset T_{n-1}(U) \subset \cdots \subset T_{1}(U)
$$

We also need a special case of a result of Poincaré and Perron (see [4, 12, 13, 14]). The difference equation (21) is called a Poincare difference equation if

$$
a_{n} \approx a n^{\alpha} \text { when } n \rightarrow \infty \text {, }
$$

where $a \neq 0, \alpha$ real. The characteristic polynomial of the Poincare difference equation is defined by

$$
\Phi(z)=z^{2}-a z-1 .
$$

LEMMA 3. (cf. $[4,12,13,14])$.

Case 1: $\alpha=0$. Let $z_{1}, z_{2}$ be the two zeros of $\Phi(z)$. Then

- If $\left|z_{1}\right|>\left|z_{2}\right|$, there exist two linearly independent solutions $c_{n, 1}$ and $c_{n, 2}$ of (21) such that

$$
\lim _{n \rightarrow \infty} \frac{c_{n+1, i}}{c_{n, i}}=z_{i}, \quad i=1,2 .
$$

- If $\left|z_{1}\right|=\left|z_{2}\right|$, then for every nontrivial solution $\left\{c_{n}\right\}$ of $(21)$

$$
\lim _{n \rightarrow \infty} \sup \left|c_{n}\right|^{1 / n}=\left|z_{1}\right| \text {. }
$$

Case 2: $\alpha>0$. There exist two linearly independent solutions $\left\{c_{n, 1}\right\}$ and $\left\{c_{n, 2}\right\}$ such that

$$
\begin{aligned}
& \frac{c_{n+1,1}}{c_{n, 1}} \approx a n^{\alpha} \quad \text { if } n \rightarrow \infty, \\
& \frac{c_{n+1,2}}{c_{n, 2}} \approx-\frac{1}{a} n^{-\alpha} \quad \text { if } n \rightarrow \infty .
\end{aligned}
$$

4. Proofs of Theorem 1 and Theorem 2. Now we give proofs for Theorem 1 and Theorem 2.

Proof of Theorem 1. Parts A, B, C mostly have been shown in Theorem 1 of [7,8] if one observes $\nu=1 / \lambda$ there and $\eta=\sigma / \lambda$ there; estimates (4) and (8) come from Eqs. (49) and (50) of [7]. From Lemma 3 of [7], we know that all the possible neutral and unstable modes will come from the case $k_{1}=1$ in Eq. (19).

Part D comes from the consideration of the case $k_{1}=1$ and $k_{2}$ odd in Eq. (19). First, we give the estimates (5), (7), and (9).

The condition for solving (19) in the case $k_{1}=1, k_{2}$ odd is

$$
a_{1}+\frac{1}{a_{2}+\frac{1}{a_{3}+\cdots}}=-i
$$


(if we require $\operatorname{Im} \eta>0$ ) where

$$
a_{n}=\frac{2 \sqrt{2} \pi\left((2 n-1)^{2}+1\right)\left(\left((2 n-1)^{2}+1\right) \nu+\eta\right)}{(2 n-1)^{2}-3}
$$

From (37), since we are interested in $\operatorname{Re} \eta \geq 0$, we see that a necessary condition for Eq. (37) is

$$
\operatorname{Re}\left(a_{2}+\frac{1}{a_{1}+i}\right)<0 .
$$

We let $\eta=\alpha+i \beta$. From (38), we substitute $a_{1}, a_{2}$ into the above inequality. After some computation, we obtain

$$
\frac{40}{3} \pi^{2}(\alpha+10 \nu)(\alpha+2 \nu)+\frac{5}{3} \frac{\alpha+10 \nu}{\alpha+2 \nu}(1-2 \sqrt{2} \pi \beta)^{2}<1 .
$$

From this we easily deduce (5), (7), and (9).

For the remainder of $D$, we give a heuristic argument below (the proof for part $\mathrm{D}$ and other related properties is quite long, the details will be given in [9]).

Define two analytic functions in $\operatorname{Re} \eta>-2 \nu$ :

$$
\begin{aligned}
& f(\eta)=a_{1}+\frac{1}{a_{2}+\frac{1}{a_{3}+\cdots}}+i, \\
& f_{2}(\eta)=a_{1}+\frac{1}{a_{2}}+i .
\end{aligned}
$$

We argue that the zeros of $f(\eta)$ have the same behavior as the zeros of $f_{2}(\eta)$. Then we solve $f_{2}(\eta)=0$ for $\operatorname{Re} \eta>-2 \nu$. We find

$$
\eta=-6 \nu+\frac{\sqrt{2}}{8 \pi} i+\frac{\sqrt{(80 \pi \nu)^{2}+\frac{35}{2}+i 400 \sqrt{2} \pi \nu}}{20 \pi} .
$$

From this, we obtain the remaining part of $D$.

Proof of Theorem 2.

Step 1. If $k_{1}=0$, then from (20) we have

$$
\eta a_{\left(0, k_{2}\right)}=0, \quad \forall k_{2}>0 .
$$

In this case there are only neutral modes, which are given by $W=W_{\left(0, k_{2}\right)}, \forall k_{2}>0$, and the corresponding eigenvalue is 0 . So there are infinitely many neutral modes corresponding to the eigenvalue 0 . In the following, we assume $k_{1}>0$.

Step 2. For each fixed $k_{1}>0$, we write (19) in the following form:

$$
a_{n} c_{n}+c_{n-1}-c_{n+1}=0, \quad n=0, \pm 1, \pm 2, \ldots,
$$

where either

$$
\begin{aligned}
& a_{n}=c_{\left(k_{1}, 2 n\right)}=\frac{2 \sqrt{2} \pi\left(k_{1}^{2}+(2 n)^{2}\right) \eta}{k_{1}\left(k_{1}^{2}+(2 n)^{2}-4\right)}, \\
& c_{n}=b_{\left(k_{1}, 2 n\right)}=\frac{k_{1}^{2}+(2 n)^{2}-4}{\sqrt{k_{1}^{2}+(2 n)^{2}}} a_{\left(k_{1}, 2 n\right)}, \quad \forall n,
\end{aligned}
$$


or

$$
\begin{aligned}
& a_{n}=c_{\left(k_{1}, 2 n-1\right)}=\frac{2 \sqrt{2} \pi\left(k_{1}^{2}+(2 n-1)^{2}\right) \eta}{k_{1}\left(k_{1}^{2}+(2 n-1)^{2}-4\right)}, \\
& c_{n}=b_{\left(k_{1}, 2 n-1\right)}=\frac{k_{1}^{2}+(2 n-1)^{2}-4}{\sqrt{k_{1}^{2}+(2 n-1)^{2}}} a_{\left(k_{1}, 2 n-1\right)}, \quad \forall n .
\end{aligned}
$$

We want to solve (40) such that either

$$
W=\sum_{n=-\infty}^{\infty} a_{\left(k_{1}, 2 n\right)} W_{\left(k_{1}, 2 n\right)}
$$

or

$$
W=\sum_{n=-\infty}^{\infty} a_{\left(k_{1}, 2 n-1\right)} W_{\left(k_{1}, 2 n-1\right)}
$$

is in the space $H$.

Step 3. We consider only half of (40):

$$
a_{n} c_{n}+c_{n-1}-c_{n+1}=0, \quad n=1,2, \ldots
$$

From (41) and (42), we see

$$
\lim _{n \rightarrow \infty} a_{n}=\frac{2 \sqrt{2} \pi \eta}{k_{1}} .
$$

So (45) is a Poincaré difference equation with $\alpha=0, a=\frac{2 \sqrt{2} \pi \eta}{k_{1}}$ as defined in Sec. 3. The corresponding characteristic polynomial is

$$
\Phi(z)=z^{2}-\frac{2 \sqrt{2} \pi \eta}{k_{1}} z-1 .
$$

Its zeros are

$$
\begin{aligned}
& z_{1}=\frac{\sqrt{2} \pi \eta}{k_{1}}+\sqrt{\frac{2 \pi^{2} \eta^{2}}{k_{1}}+1} \\
& z_{2}=\frac{\sqrt{2} \pi \eta}{k_{1}}-\sqrt{\frac{2 \pi^{2} \eta^{2}}{k_{1}}+1}
\end{aligned}
$$

It is easy to show:

- If $\operatorname{Re} \eta>0$, then $\left|z_{1}\right|>1,\left|z_{2}\right|<1$.

- If $\operatorname{Re} \eta=0$ and $|\eta| \leq \frac{k_{1}}{\sqrt{2} \pi}$, then $\left|z_{1}\right|=\left|z_{2}\right|=1$.

- If $\operatorname{Re} \eta=0$ and $|\eta|>\frac{k_{1}}{\sqrt{2} \pi}$, then

1. if $\operatorname{Im} \eta>\frac{k_{1}}{\sqrt{2} \pi},\left|z_{1}\right|>1,\left|z_{2}\right|<1$;

2. if $\operatorname{Im} \eta<\frac{k_{1}}{\sqrt{2} \pi},\left|z_{1}\right|<1,\left|z_{2}\right|>1$.

Now using Lemma 3, we obtain that if $\operatorname{Re} \eta>0$ or $\operatorname{Re} \eta=0,|\eta|>\frac{k_{1}}{\sqrt{2} \pi}$, then there exist solutions of (45) that grow exponentially with $n$ as $n \rightarrow \infty$. This implies that there are solutions of (40) that grow exponentially with $n$ as $n \rightarrow \infty$. Similarly one can see that there are solutions of (40) that grow exponentially with $n$ as $n \rightarrow-\infty$. 
Combining these two facts, we imply that there is a solution of (40) that grows exponentially with $n$ as $|n| \rightarrow \infty$. By (41), (42), (43), and (44), we obtain that a necessary condition for $W$ to be in the space $H$ is that the corresponding $\left\{c_{n}\right\}$ is a minimal solution for (40).

Step 4. We consider the case $\operatorname{Re} \eta>0$ and $\operatorname{Re} \eta=0,|\eta| \geq \frac{k_{1}}{\sqrt{2} \pi}$ for each fixed $k_{1}=1,2, \ldots$ in Step 5 and Step 6 below. We first try to determine all the minimal solutions by using Lemma 1 and then show the minimal solution we get corresponding to an eigenvector by using either Lemma 2 or Lemma 3. By Lemma 1 , there is a minimal solution $\left\{c_{n}\right\}$ for (40) if and only if

$$
\frac{c_{1}}{c_{0}}=a_{0}+\frac{1}{a_{-1}+\frac{1}{a_{-2}+\cdots}}=\frac{-1}{a_{1}+\frac{1}{a_{2}+\cdots}} .
$$

Step 5. The case $\operatorname{Re} \eta=0,|\eta| \geq \frac{k_{1}}{\sqrt{2} \pi}$ for fixed $k_{1}=1,2, \ldots$.

If $k_{2}$ is odd, that is the case (40) and (42). Since now $a_{n}=a_{-(n-1)}$, Eq. (48) becomes

$$
\pm i=a_{1}+\frac{1}{a_{2}+\frac{1}{a_{3}+\cdots}} .
$$

Since $\left|a_{1}\right| \geq 2,\left|a_{n}\right|>2, \forall n \geq 2$, by Theorem 4.35 of [5]

$$
\mid \text { RHS of }(49)|\geq| a_{1}\left|-\frac{1}{\left|a_{2}\right|-\frac{1}{\left|a_{3}+\cdots\right|}}>2-1=1=\right| \text { LHS of }(49) \mid \text {, }
$$

which makes (49) impossible. Thus there are no neutral modes in this case.

If $k_{2}$ is even, that is the case (40) and (41). Since $a_{n}=a_{-n}$, Eq. (48) becomes

$$
\frac{1}{a_{1}+\frac{1}{a_{2}+\cdots}}=-\frac{a_{0}}{2}
$$

First, if $k_{1}=2$, by (41), we have

$$
a_{0}=\infty, \quad\left|a_{1}\right| \geq 4, \quad\left|a_{n}\right|>2, \quad \forall n \geq 2 .
$$

But from (48), we get

$$
a_{1}+\frac{1}{a_{2}+\frac{1}{a_{3}+\cdots}}=0
$$

which is impossible because of (51) and Theorem 4.35 of [5]. Hence (50) is impossible and there are no neutral modes in this case either.

Secondly, if $k_{1} \geq 3$, by (41), we get

$$
\left|a_{n}\right|>2, \quad \forall n .
$$

So the magnitude of the right-hand side of $(50)$ is larger than 1 , but by Theorem 4.35 of [5], we see that the magnitude of the left-hand side of (50) is less than 1 , hence (50) is impossible. So there are no neutral modes in this case.

Thirdly, if $k_{1}=1$, from (41), we have

$$
\left|a_{n}\right|>2, \quad \forall n \geq 2 \text { and }\left|a_{1}\right| \geq 10 \text { and }\left|a_{0}\right| \geq 2 / 3 \text {. }
$$


Hence, the right-hand side of $(50)$ satisfies

$$
\mid \text { RHS } \mid>1 / 3 \text {. }
$$

But by Theorem 4.35 of [5], the left-hand side of (50) satisfies

$$
\begin{aligned}
\mid \text { LHS } \mid & \leq \frac{1}{\left|a_{1}\right|-\left|\frac{1}{a_{2}+\cdots}\right|} \\
& <\frac{1}{10-1}=\frac{1}{9} .
\end{aligned}
$$

Inequalities (52) and (53) imply that (50) is impossible; so there is no neutral mode either.

Step 6. Now we consider the case of unstable modes, the case $\operatorname{Re} \eta>0$.

If $\operatorname{Re} \eta>0$, by a proof similar to Lemma 3 in [7], we obtain that if $k_{1} \geq 2$, then (48) is impossible. So the possible unstable modes will come from the case $k_{1}=1$.

If $k_{1}=1$ and $k_{2}$ is odd, that is the situation of (42). Since now $a_{n}=a_{-(n-1)}$, (48) becomes

$$
a_{1}+\frac{1}{a_{2}+\frac{1}{a_{3}+\cdots}}= \pm i,
$$

where

$$
a_{n}=\frac{2 \sqrt{2} \pi\left((2 n-1)^{2}+1\right) \eta}{(2 n-1)^{2}-3} .
$$

Since $a_{n}=a_{-(n-1)}$, by the uniqueness of minimal solution (within a constant factor), it can be seen that the possible minimal solution $\left\{c_{n}\right\}$ satisfies $c_{n}= \pm i(-1)^{n} c_{-(n-1)}$. We want to solve (54) for $\operatorname{Re} \eta>0$. This will be done in Lemma 6 below. Also Lemma 6 gives the inequalities (11) and (12). The reason our minimal solution obtained from Lemma 6 corresponds to an eigenvector is due to Lemma 3 (from which we see that the minimal solution decreases exponentially fast; see discussions in Sec. 5). We get part B.

If $k_{1}=1$ and $k_{2}$ is even, that is the case of (41). We show there is a unique $\eta_{0}$ that satisfies Theorem 2 and the corresponding eigenspace is two dimensional.

Since $a_{n}=a_{-n},(48)$ becomes

$$
\frac{1}{a_{1}+\frac{1}{a_{2}+\cdots}}=-\frac{a_{0}}{2}
$$

where

$$
a_{n}=\frac{2 \sqrt{2} \pi\left(4 n^{2}+1\right) \eta}{4 n^{2}-3}
$$

Equation (55) can be written as

$$
\frac{1}{\eta a_{1}+\frac{1}{\eta^{-1} a_{2}+\cdots}}=\frac{\sqrt{2} \pi}{3} .
$$

Since $a_{n}=a_{-n}$, it is easy to see that the possible minimal solution $\left\{c_{n}\right\}$ satisfies $c_{n}=(-1)^{n} c_{-n}$. 
We want to solve (56) for $\operatorname{Re} \eta>0$. It can be seen that it is necessary for $\eta$ to be a real number. Define the left-hand side of the above equation as $f(\eta)$. Because

$$
\frac{1}{\eta a_{1}+\frac{1}{\eta^{-1} a_{2}}}<f(\eta)<\frac{1}{\eta a_{1}},
$$

so

hence

$$
\frac{1}{10 \sqrt{2} \pi \eta^{2}+\frac{13}{34 \sqrt{2} \pi}}<f(\eta)<\frac{1}{10 \sqrt{2} \pi \eta^{2}}
$$

$$
\begin{aligned}
& \lim _{\eta \rightarrow \infty} f(\eta)=0, \\
& \lim _{\eta \rightarrow 0} f(\eta)>\frac{34 \sqrt{2} \pi}{12} .
\end{aligned}
$$

From (58) and (59), we get that there is an $\eta_{0}>0$ such that

$$
f\left(\eta_{0}\right)=\frac{\sqrt{2} \pi}{3}
$$

that is, (56) is satisfied by this $\eta_{0}$. Because $\eta a_{2 n-1}$ increases monotonically with $\eta$, and $\eta^{-1} a_{2 n}$ is independent of $\eta$, so $f(\eta)$ is a monotonically decreasing function with respect to $\eta>0$; hence the above $\eta_{0}$ is unique. That the corresponding eigenspace is two dimensional follows from the fact that even and odd eigenvectors each contribute one.

Now we give an estimate for $\eta_{0}$. Substituting $\eta$ by $\eta_{0}$ and $f(\eta)$ by $\frac{\sqrt{2} \pi}{3}$ in (57) and simplifying, we obtain

$$
\sqrt{\frac{89}{34 \cdot 20}} \frac{1}{\pi}<\eta_{0}<\sqrt{\frac{3}{20}} \frac{1}{\pi}
$$

which gives (10) of Theorem 2.

Now we show that the minimal solution $\left\{c_{n}\right\}$ we get corresponds to an eigenvector, that is, we want to show that $W$ is in the space $H$. This can be done either from Lemma 2 or from Lemma 3. We use Lemma 2 below. From (41) and (43),

$$
W=\sum_{-\infty}^{\infty} \frac{\sqrt{4 n^{2}+1}}{4 n^{2}-3} c_{n} W_{(1,2 n)}
$$

So we only need to show the minimal solution $\left\{c_{n}\right\}$ is bounded. This is proved by noting

$$
\begin{aligned}
\operatorname{Re} a_{n} & >0, \quad \forall n \neq 0, \\
a_{n} & \approx 2 \sqrt{2} \pi \eta_{0} \\
& >2 \sqrt{2} \pi \sqrt{\frac{89}{34 \cdot 20}} \frac{1}{\pi} \\
& =\sqrt{\frac{89}{85}} \\
& >1 .
\end{aligned}
$$


So the conditions for Lemma 2 are satisfied; thus we obtain

$$
\lim _{|n| \rightarrow \infty} c_{n}=0 .
$$

Step 7. The case $\operatorname{Re} \eta=0,|\eta|<\frac{k_{1}}{\sqrt{2} \pi}$ for each fixed $k_{1}=1,2, \ldots$. From Step 3 , the zeros $z_{1}, z_{2}$ for the characteristic polynomial are given by (47) and satisfy

$$
z_{1} \neq z_{2}, \quad\left|z_{1}\right|=\left|z_{2}\right|=1
$$

From (41) and (42), we obtain

$$
a_{n}=\frac{2 \sqrt{2} \pi \eta}{k_{1}}+O\left(\frac{1}{n^{2}}\right)
$$

hence, the conditions for Lemma 4 below are satisfied with

$$
\begin{array}{ll}
a=-\frac{2 \sqrt{2} \pi \eta}{k_{1}}, & b=-1, \\
x_{n}=O\left(\frac{1}{n^{2}}\right), & y_{n}=0 .
\end{array}
$$

So by Lemma 4 below, we obtain that all the solutions of (40) are bounded. So all these $\eta$ are neutral modes and the corresponding eigenspace is infinite dimensional.

We have proved Theorem 2.

5. Some lemmas and proof of Theorem 3. Now we give some lemmas. Consider the following Poincare difference equation $[12,13,14]$ :

$$
c_{n+2}+\left(a+x_{n}\right) c_{n+1}+\left(b+y_{n}\right) c_{n}=0, \quad n=1,2, \ldots,
$$

where

$$
x_{n} \rightarrow 0, \quad y_{n} \rightarrow 0 \text { if } n \rightarrow \infty .
$$

The corresponding characteristic polynomial is

$$
\Phi(z)=z^{2}+a z+b=\left(z-z_{1}\right)\left(z-z_{2}\right) .
$$

Lemma 4. Assume $z_{1} \neq z_{2},\left|z_{1}\right| \leq 1,\left|z_{2}\right| \leq 1$ and

$$
\begin{aligned}
& \sum_{n=1}^{\infty}\left|x_{n}\right|<\infty, \\
& \sum_{n=1}^{\infty}\left|y_{n}\right|<\infty .
\end{aligned}
$$

Then all the solutions of (61) are bounded.

Proof. For each $n=1,2, \ldots$, let

$$
\begin{aligned}
c_{n} & =p_{1}(n)+p_{2}(n), \\
c_{n+1} & =z_{1} p_{1}(n)+z_{2} p_{2}(n) .
\end{aligned}
$$

Since $z_{1} \neq z_{2}, p_{1}(n)$ and $p_{2}(n)$ are uniquely determined. 
From (65) and (66), we get

$$
-z_{1} c_{n}+c_{n+1}=-\left(z_{1}-z_{2}\right) p_{2}(n)=\Phi^{\prime}\left(z_{2}\right) p_{2}(n) .
$$

So from (61), (62), (65), (66), and (67)

$$
\begin{aligned}
\Phi^{\prime}\left(z_{2}\right) p_{2}(n+1)= & -z_{1} c_{n+1}+c_{n+2} \\
= & -z_{1} c_{n+1}-\left(a+x_{n}\right) c_{n+1}-\left(b+y_{n}\right) c_{n} \\
= & \left(-z_{1}-a\right) c_{n+1}-b c_{n}-x_{n} c_{n+1}-y_{n} c_{n} \\
= & z_{2} c_{n+1}-z_{1} z_{2} c_{n}-x_{n} c_{n+1}-y_{n} c_{n} \\
= & z_{2}\left(-z_{1} c_{n}+c_{n+1}\right)-x_{n}\left(z_{1} p_{1}(n)+z_{2} p_{2}(n)\right) \\
& -y_{n}\left(p_{1}(n)+p_{2}(n)\right) ;
\end{aligned}
$$

hence,

$$
\boldsymbol{\Phi}^{\prime}\left(z_{2}\right) p_{2}(n+1)=z_{2} \Phi^{\prime}\left(z_{2}\right) p_{2}(n)-X_{1}(n) p_{1}(n)-X_{2}(n) p_{2}(n)
$$

where

$$
X_{1}(n)=z_{1} x_{n}+y_{n}, \quad X_{2}(n)=z_{2} x_{n}+y_{n} .
$$

From (68), we obtain

$$
p_{2}(n+1)=z_{2} p_{2}(n)-\alpha_{2}(n) p_{1}(n)-\beta_{2}(n) p_{2}(n) ;
$$

similarly, we have

$$
p_{1}(n+1)=z_{1} p_{1}(n)-\alpha_{1}(n) p_{1}(n)-\beta_{1}(n) p_{2}(n),
$$

where

$$
\alpha_{i}(n)=\frac{X_{1}(n)}{\Phi^{\prime}\left(z_{i}\right)}, \quad \beta_{i}(n)=\frac{X_{2}(n)}{\Phi^{\prime}\left(z_{i}\right)}, \quad i=1,2 .
$$

We write (70) and (71) in the matrix form

$$
\left(\begin{array}{l}
p_{1}(n+1) \\
p_{2}(n+1)
\end{array}\right)=A(n)\left(\begin{array}{l}
p_{1}(n) \\
p_{2}(n)
\end{array}\right)
$$

where

$$
A(n)=\left(\begin{array}{cc}
z_{1}-\alpha_{1}(n) & -\beta_{1}(n) \\
-\alpha_{2}(n) & z_{2}-\beta_{2}(n)
\end{array}\right) .
$$

So

$$
\left(\begin{array}{l}
p_{1}(n+1) \\
p_{2}(n+1)
\end{array}\right)=A(n) A(n-1) \cdots A(1)\left(\begin{array}{l}
p_{1}(1) \\
p_{2}(1)
\end{array}\right) \text {. }
$$

The matrix operator $A(n)$ acts from $C^{2}$ to $C^{2}$. Since $\left|z_{1}\right|=\left|z_{2}\right| \leq 1$, its norm satisfies

$$
\begin{aligned}
\|A(n)\| & \leq \max \left\{\left|z_{1}-\alpha_{1}(n)\right|+\left|\beta_{1}(n)\right|,\left|\alpha_{2}(n)\right|+\left|z_{2}-\beta_{2}(n)\right|\right\} \\
& \leq \max \left\{1+\left|\alpha_{1}(n)\right|+\left|\beta_{1}(n)\right|, 1+\left|\alpha_{2}(n)\right|+\left|\beta_{2}(n)\right|\right\} \\
& \leq 1+\gamma_{n},
\end{aligned}
$$

where

$$
\gamma(n)=\left|\alpha_{1}(n)\right|+\left|\beta_{1}(n)\right|+\left|\alpha_{2}(n)\right|+\left|\beta_{2}(n)\right|
$$


By the assumptions (63) and (64), we see

$$
\sum_{n=1}^{\infty} \gamma(n)<\infty
$$

Hence from (72), (73), and (74), we get

$$
\begin{aligned}
\max \left\{\left|p_{1}(n+1)\right|,\left|p_{2}(n+1)\right|\right\} & \leq \prod_{k=1}^{k=n}(1+\gamma(k)) \max \left\{\left|p_{1}(1)\right|,\left|p_{2}(1)\right|\right\} \\
& \leq e^{\sum_{k=1}^{k=n} \gamma(k)} \max \left\{\left|p_{1}(1)\right|,\left|p_{2}(1)\right|\right\} \\
& <e^{\sum_{k=1}^{k=\infty} \gamma(k)} \max \left\{\left|p_{1}(1)\right|,\left|p_{2}(1)\right|\right\}
\end{aligned}
$$

So all the solutions of (61) are bounded.

Now we discuss the unstable eigenvector. From previous discussions, the only unstable mode for both the Euler equations and the Navier-Stokes equations is given by $k_{1}=1, k_{2}$ even or odd. We rewrite the determining equation (in the even case) as follows (the odd case is similar):

$$
\begin{aligned}
& a_{n} c_{n}+c_{n-1}=c_{n+1}, \quad n=0, \pm 1, \pm 2, \ldots, \\
& \frac{1}{a_{1}+\frac{1}{a_{2}+\cdots}}=-\frac{a_{0}}{2},
\end{aligned}
$$

where

$$
a_{n}=\frac{2 \sqrt{2} \pi\left(4 n^{2}+1\right)\left(\nu\left(4 n^{2}+1\right)+\eta\right)}{4 n^{2}-3}
$$

Our unstable mode is given by

$$
W=\sum_{-\infty}^{\infty} \frac{\sqrt{4 n^{2}+1}}{4 n^{2}-3} c_{n} W_{(1,2 n)},
$$

where $\left\{c_{n}\right\}$ is generated by (76) with $c_{0}=1, c_{1}=a_{0} / 2$.

If $\nu>0$, we see that the conditions of Lemma 3 are satisfied with $\alpha=2$ and $a=8 \sqrt{2} \pi \nu$. So by Lemma 3 , our solution $\left\{c_{n}\right\}$ satisfies

so

$$
\frac{c_{n+1}}{c_{n}} \approx-\frac{1}{8 \sqrt{2} \pi \nu} n^{-2} \text { as }|n| \rightarrow \infty ;
$$

$$
\frac{a_{(1,2 n+2)}}{a_{(1,2 n)}} \approx-\frac{n}{n+1} \frac{1}{8 \sqrt{2} \pi \nu} n^{-2} \text { as }|n| \rightarrow \infty .
$$

Hence, in determination of the unstable mode, the low harmonic is more important than the high harmonic, because we see from $(80)$ that the magnitude of the high harmonic decreases very fast.

If $\nu=0$, the conditions of Lemma 3 are satisfied with $\alpha=0$ and $z_{1}, z_{2}$ given by (47) with $k_{1}=1,\left|z_{1}\right|>\left|z_{2}\right|$. Hence for our unstable mode,

$$
\lim _{|n| \rightarrow \infty} \frac{a_{(1,2 n+2)}}{a_{(1,2 n)}}=z_{2} \text {. }
$$


Since $\left|z_{2}\right|<1$, the high harmonic decreases exponentially but much slower as compared with the viscous case. We also get here that the unstable modes are infinitely smooth functions.

Lemma 5. For the three-term recurrence relations (22), if all the solutions are bounded, then

- there is no nontrivial solution $\left\{c_{n}\right\}$ such that

$$
\lim _{|n| \rightarrow \infty} c_{n}=0 \text {, }
$$

- there is no minimal solution.

Proof. By assumption all the solutions of (22) are bounded; so there is a constant $M$ such that, for any solution $\left\{c_{n}\right\}$ of $(22)$,

$$
\left|c_{n}\right| \leq M, \quad \forall n .
$$

Suppose there is a solution $\left\{c_{n}\right\}$ such that

$$
\lim _{|n| \rightarrow \infty} c_{n}=0 .
$$

Let $\left\{d_{n}\right\}$ be another solution of (22) that is linearly independent of $\left\{c_{n}\right\}$. Define

$$
D(n)=\left|\begin{array}{cc}
c_{n} & d_{n} \\
c_{n+1} & d_{n+1}
\end{array}\right| .
$$

For every $n$, it is easy to see

$$
D(n)=(-1)^{n} D(0) .
$$

Since $\left\{c_{n}\right\}$ and $\left\{d_{n}\right\}$ are linearly independent, we have

$$
|D(n)|=|D(0)| \neq 0, \quad \forall n \geq 0 .
$$

But,

$$
\begin{aligned}
|D(n)| & =\left|c_{n} d_{n+1}-d_{n} c_{n+1}\right| \\
& \leq M\left(\left|c_{n}\right|+\left|c_{n+1}\right|\right) \\
& \rightarrow 0 \text { if } n \rightarrow \infty,
\end{aligned}
$$

which is a contradiction with (83). We have proved the first part.

Now if there is a minimal solution $\left\{c_{n}\right\}$, then there is another solution $\left\{d_{n}\right\}$ such that

$$
\lim _{|n| \rightarrow \infty} \frac{c_{n}}{d_{n}}=0 .
$$

But

$$
\frac{\left|c_{n}\right|}{M} \leq\left|\frac{c_{n}}{d_{n}}\right|, \quad \forall n
$$

so

$$
\lim _{|n| \rightarrow \infty} c_{n}=c,
$$

which is impossible from the above. Thus, we have proved the second part. 
LEMMA 6. For the equation

$$
a_{1}+\frac{1}{a_{2}+\frac{1}{a_{3}+\cdots}}=-i
$$

where

$$
a_{n}=\frac{2 \sqrt{2} \pi\left((2 n-1)^{2}+1\right) \eta}{(2 n-1)^{2}-3},
$$

there is a unique solution $\eta$ with $\operatorname{Re} \eta>0$. If we let $\eta=\alpha+i \beta$, then

$$
\alpha^{2}+\left(\beta-\frac{1}{2 \sqrt{2} \pi}\right)^{2}<\frac{3}{40 \pi^{2}}
$$

Proof. Define

$$
\begin{gathered}
f(\eta)=a_{1}+\frac{1}{a_{2}+\frac{1}{a_{3}+\cdots}}+i, \\
f_{2}(\eta)=a_{1}+\frac{1}{a_{2}}+i .
\end{gathered}
$$

Both functions are analytic functions for $\eta>0$ (see [5]).

Solving

$$
f_{2}(\eta)=0 \text {, }
$$

we obtain

Let

$$
\eta= \pm \frac{\sqrt{70}}{40 \pi}+\frac{5 \sqrt{2}}{40 \pi} i
$$

$$
D=\left\{\eta|\operatorname{Re} \eta>\varepsilon,| \eta \mid \leq \frac{r}{\sqrt{2} \pi}\right\}
$$

where $\varepsilon$ is a small positive number and $r \geq 1$.

We want to show

$$
\left|f(\eta)-f_{2}(\eta)\right|<|f(\eta)|+\left|f_{2}(\eta)\right| \text { for } \eta \in \partial D .
$$

Then we can apply Rouché's Theorem.

We note that if (91) is not true, then there is an $\eta \in \partial D$ and a $\delta \quad(0 \leq \delta \leq \infty)$ such that

So

$$
f(\eta)=-\delta f_{2}(\eta)
$$

$$
a_{1}+i+\frac{\frac{1}{1+\delta}}{a_{2}+\frac{1}{a_{3}+\cdots}}+\frac{\delta}{1+\delta} \frac{1}{a_{2}}=0 .
$$

First, we show that for all $\delta: 0 \leq \delta \leq \infty$, (92) is not satisfied by any $\eta$ with

If $\eta$ satisfies (93), from (85)

$$
|\eta| \geq \frac{1}{\sqrt{2} \pi}
$$

$$
\begin{aligned}
& \left|a_{1}\right| \geq 2, \\
& \left|a_{2}\right| \geq \frac{10}{3}, \\
& \left|a_{3}\right| \geq \frac{26}{11} .
\end{aligned}
$$


So by Theorem 4.35 of [5], we get

$$
\begin{aligned}
\left|\frac{\frac{1}{1+\delta}}{a_{2}+\frac{1}{a_{3}+\cdots}}\right| & \leq \frac{\frac{1}{1+\delta}}{\left|a_{2}\right|-\frac{1}{\left|a_{3}+\cdots\right|}} \\
& \leq \frac{\frac{1}{1+\delta}}{\frac{10}{3}-\frac{1}{26 / 11-1}} \\
& =\frac{1}{1+\delta} \frac{15}{39} \\
& \leq \frac{15}{39} .
\end{aligned}
$$

But

$$
\begin{aligned}
\left|a_{1}+i+\frac{\delta}{1+\delta} \frac{1}{a_{2}}\right| & \geq\left|a_{1}\right|-1-\frac{\delta}{1+\delta} \frac{1}{\left|a_{2}\right|} \\
& \geq 1-\frac{\delta}{1+\delta} \frac{3}{10} \\
& \geq 1-\frac{3}{10} \\
& \geq \frac{7}{10} .
\end{aligned}
$$

From (95) and (96), we readily obtain that (92) is impossible.

Secondly, we show that for all $\delta: 0 \leq \delta \leq \infty$, there is an $\varepsilon_{0}>0$ such that Eq. (92) is not satisfied by any $\eta$ with $0<\operatorname{Re} \eta<\varepsilon_{0}$. If this is not the case then there are $\delta_{j}, \eta_{j}, j=1,2, \ldots$,

$$
\begin{gathered}
0 \leq \delta_{j} \leq \infty, \\
\operatorname{Re} \eta_{j}>0 \text { and } \lim _{j \rightarrow \infty} \operatorname{Re} \eta_{j}=0,
\end{gathered}
$$

such that (92) is satisfied for each pair $\delta_{j}, \eta_{j}$. By the above consideration, we see that $\eta_{j}$ has to be bounded $\left(\left|\eta_{j}\right|<\frac{1}{\sqrt{2} \pi}, \forall j\right)$; hence, there is a subsequence (still denoted by $\eta_{j}$ ) such that

$$
\lim _{j \rightarrow \infty} \eta_{j}=\eta_{0} \quad \text { and } \quad \operatorname{Re} \eta_{0}=0
$$

If $\delta_{j}$ is unbounded, there is a subsequence (still denoted by $\delta_{j}$ ) such that

$$
\lim _{j \rightarrow \infty} \delta_{j}=\infty
$$

thus, from (92) we have

$$
a_{1}+i+\frac{1}{a_{2}} \approx 0 \text { if } j \rightarrow \infty,
$$

which is impossible from (89). Hence, $\delta_{j}$ has to be bounded. So, there is a subsequence of $\delta_{j}$ (still denoted by $\delta_{j}$ ) that converges to a finite number $\delta_{0}$,

$$
\lim _{j \rightarrow \infty} \delta_{j}=\delta_{0} .
$$


By (92), (97), and (98), we have

$$
a_{1}\left(\eta_{0}\right)+i+\frac{\frac{1}{1+\delta_{0}}}{a_{2}\left(\eta_{0}\right)+\frac{1}{a_{3}\left(\eta_{0}\right)+\cdots}}+\frac{\delta_{0}}{1+\delta_{0}} \frac{1}{a_{2}\left(\eta_{0}\right)} \approx 0 .
$$

From the consideration above, we note that $\eta_{0}$ has to satisfy

$$
\left|\eta_{0}\right|<\frac{1}{\sqrt{2} \pi}
$$

But for these $\eta_{0}$,

$$
\frac{1}{a_{2}\left(\eta_{0}\right)+\frac{1}{a_{3}\left(\eta_{0}\right)+\cdots}}
$$

diverges (this is from Step 7 of the proof for Theorem 2 and Lemma 5; there is no minimal solution in this case); so (99) is impossible, hence a contradiction.

We have proved there is an $\varepsilon_{0}>0$ such that for any $\varepsilon \quad\left(0<\varepsilon<\varepsilon_{0}\right)$ and any $r \geq 1,(91)$ is true. From (89), $f_{2}(\eta)$ has only one zero in $\operatorname{Re} \eta>0$. By Rouché's Theorem, we get that there is a unique solution $\eta$ of (84) with $\operatorname{Re} \eta>0$.

The estimate $(86)$ is from the observation that a necessary condition for $(84)$ is

$$
\operatorname{Re}\left(a_{2}+\frac{1}{a_{1}+i}\right)<0 .
$$

We substitute $a_{1}, a_{2}$ into this and, simplifying, we obtain (86). We are done.

Finally, we prove Theorem 3.

Proof of Theorem 3. We first show (13) of Theorem 3. As we noted before, $\eta(\nu)$ and $\eta_{0}$ come from the case $k_{1}=1, k_{2}$ even, that is, they are given by the solutions of (76), (77), and (78). Define

$$
f(\nu, \eta)=\frac{a_{0}}{2}+\frac{1}{a_{1}+\frac{1}{a_{2}+\cdots}}
$$

and

$$
f_{n}(\nu, \eta)=\frac{a_{0}}{2}+\frac{1}{a_{1}+\frac{1}{a_{2}+\cdots+\frac{1}{a_{n}}}} .
$$

From part $\mathrm{C}$ of Theorem 1 and part $\mathrm{A}$ of Theorem 2

$$
\begin{aligned}
f(\nu, \eta(\nu)) & =0, \quad \forall \nu>0, \\
f\left(0, \eta_{0}\right) & =0 .
\end{aligned}
$$

Also, there are positive constants $\delta_{0}, c_{1}, c_{2}\left(c_{1}<c_{2}\right)$ such that

$$
\begin{gathered}
c_{1} \leq \eta_{0} \leq c_{2}, \\
c_{1} \leq \eta(\nu) \leq c_{2} \quad \text { when } 0<\nu \leq \delta_{0} .
\end{gathered}
$$

Hence, if (13) is not true, there exist subsequences $\nu_{k}$ and $\eta\left(\nu_{k}\right)$ such that

$$
\lim _{\nu_{k} \rightarrow 0} \eta\left(\nu_{k}\right)=\eta_{0}^{\prime}
$$

where

$$
\eta_{0}^{\prime} \neq \eta_{0} \quad \text { and } \quad c_{1} \leq \eta_{0}^{\prime} \leq c_{2}
$$


Now from properties of continued fractions ([5]), we see that

$$
\lim _{n \rightarrow \infty} f_{n}(\nu, \eta)=f(\nu, \eta)
$$

uniformly for

$$
c_{1} \leq \eta \leq c_{2} \text { and } 0 \leq \nu \leq \delta_{0} .
$$

So for any $\varepsilon>0$, there is an integer $N$ such that if $n \geq N$, then for any $\nu, \eta$ satisfying condition (107)

$$
\left|f_{n}(\nu, \eta)-f(\nu, \eta)\right|<\varepsilon .
$$

Now we put $\nu=\nu_{k}, \eta(\nu)=\eta\left(\nu_{k}\right)$ into the above equation. By (103), we get

$$
\left|f_{n}\left(\nu_{k}, \eta\left(\nu_{k}\right)\right)\right|<\varepsilon .
$$

For fixed $n \geq N$, we let $\nu_{k} \rightarrow 0$ in (108). Because of (105), we get

$$
\left|f_{n}\left(0, \eta_{0}^{\prime}\right)\right| \leq \varepsilon
$$

hence,

$$
\limsup _{n \rightarrow \infty}\left|f_{n}\left(0, \eta_{0}^{\prime}\right)\right| \leq \varepsilon
$$

But from (106)

$$
\lim _{n \rightarrow \infty}\left|f_{n}\left(0, \eta_{0}^{\prime}\right)\right|=\left|f\left(0, \eta_{0}^{\prime}\right)\right|
$$

so

$$
\left|f\left(0, \eta_{0}^{\prime}\right)\right| \leq \varepsilon
$$

Because $\varepsilon$ can be arbitrarily small, thus

$$
\left|f\left(0, \eta_{0}^{\prime}\right)\right|=0 .
$$

By part A of Theorem 2, from (104) and (109), we get a contradiction; so (13) has to be true. [9])

By the fact that there are positive constants $c_{1}, c_{2}$ such that (this will be given in

$$
c_{1} \leq \operatorname{Re} \eta_{1}(\nu) \leq c_{2} \text { when } \nu \rightarrow 0,
$$

one readily proves (14) of Theorem 3 similarly as above.

6. On a result of Vishik and Friedlander. Now consider a general steady flow $u_{0}$. For the operator $L_{\nu}, \nu \geq 0$ of (1) and (2) we define

$$
\sigma_{\nu}=\max \left\{\operatorname{Re} \eta \mid \eta \text { is an eigenvalue of } L_{\nu}\right\} .
$$

In [18], by using dynamo theory, Vishik and Friedlander proved the following result. THEOREM.

$$
\varlimsup_{\nu \rightarrow 0} \sigma_{\nu} \leq \sigma_{0} .
$$

REMARK. It is called "fast vorticity generation" if

$$
\varlimsup_{\nu \rightarrow 0} \sigma_{\nu}>0 \text {. }
$$


From Theorem 3, we see in certain cases, not only "fast vorticity generation" is possible, but one actually has

$$
\varlimsup_{\nu \rightarrow 0} \sigma_{\nu}=\sigma_{0} .
$$

From this, one might wonder whether the equality in (111) is true for general steady flow, but the following simple example shows this is not the case in general.

Now, we give an example with instability in the Euler equations but the corresponding Navier-Stokes equations are stable for all Reynolds numbers, that is, there is no vorticity generation at all.

EXAMPLE. Let

$$
u_{0}=\left(\begin{array}{l}
A_{1} \sin x_{2}+B_{1} \cos x_{2} \\
A_{2} \sin x_{1}+B_{2} \cos x_{1}
\end{array}\right),
$$

where $A_{1}, B_{1}, A_{2}, B_{2}$ are constants, and $A_{1}^{2}+B_{1}^{2} \neq 0, A_{2}^{2}+B_{2}^{2} \neq 0$.

It is easy to see that the steady flow $u_{0}$ given above has a hyperbolic stagnation point 0 (a point $x_{0}$ is a hyperbolic stagnation point of the flow $u_{0}$ if $u_{0}\left(x_{0}\right)=0$, and the spectrum of the matrix $\frac{\partial u_{0}}{\partial x}$ at $x_{0}$ has empty intersection with the imaginary axis); so the corresponding Euler equation is locally unstable [18, 3]. Now from [10] (see also [2]), the corresponding Navier-Stokes equation is globally stable for any Reynolds numbers; so no vorticity generation is possible. Thus for this steady flow $u_{0}$, we have

$$
\begin{aligned}
& \sigma_{0}>0, \\
& \sigma_{\nu} \leq 0 \text { for } \forall \nu>0 .
\end{aligned}
$$

Acknowledgment. The author thanks Professor J. B. Keller and Professor T. P. Liu for valuable comments. The author also thanks Professor B. Rozovskii (USC) for giving the author the preprints $[3,18]$.

\section{REFERENCES}

[1] P. Constantin and C. Foias, Navier-Stokes Equations, the University of Chicago Press, Chicago, 1988

[2] P. Constantin, C. Foias, and R. Temam, On the dimension of the attractors in two-dimensional turbulence, Physica D. 30, 284-296 (1988)

[3] S. Friedlander and M. M. Vishik, Instability criteria for the flow of an inviscid incompressible fluid, Phys. Rev. Lett. 66, no. 17

[4] W. Gautschi, Computational aspects of three-term recurrence relations, SIAM Review 9 (Jan., 1967)

[5] W. B. Jones and W. J. Thron, Continued fractions, analytic theory and applications, Encyclopedia of Math. and Its Appl. 11 (1980)

[6] C. C. Lin, The Theory of Hydrodynamic Stability, Cambridge University Press, Cambridge, 1964

[7] V. X. Liu, An example of instability for the Navier-Stokes equations on the 2-dimensional torus, Comm. Partial Differential Equations (to appear)

[8] V. X. Liu, Instability for the Navier-Stokes equations on the 2-dimensional torus and a lower bound for the Hausdorff dimension of their global attractors, Comm. Math. Phys. 147, 217-230 (1992)

[9] V. X. Liu, On bifurcations of the Navier-Stokes equations on the 2D torus, in preparation

[10] C. Marchioro, An example of absence of turbulence for any Reynolds number, Comm. Math. Phys. 105, 99-106 (1986)

[11] L. D. Meshalkin and Ya. G. Sinai, Investigation of the stability of a stationary solution of a system of equations for the plane movement of an incompressible viscous fluid, J. Appl. Math. Mech. 25 (1961) 
[12] L. M. Milne-Thomson, The Calculus of Finite Differences, Macmillan, London, 1933

[13] O. Perron, Uber linear Differenzengleichungen, Acta Math. 34, 109-137 (1911)

[14] H. Poincaré, Sur les équations linéaires aux différentielles ordinaires et aux différences finies, Amer. J. Math. 7, 203-258 (1885)

[15] D. H. Sattinger, The mathematical problem of hydrodynamic stability, J. Math. Mech. 19 (1970)

[16] R. Temam, Navier-Stokes Equations and Nonlinear Functional Analysis, CBMS-NSF Regional Conference Series in Applied Math. 41, SIAM, Philadelphia, 1983

[17] R. Temam, Navier-Stokes Equations. Theory and Numerical Analysis, North-Holland Publishing Co., Amsterdam, New York, 1984

[18] M. M. Vishik and S. Friedlander, Dynamo theory methods for hydrodynamic stability, J. Math. Pure Appl. (to appear)

[19] V. I. Yudovich, Example of the generation of a secondary stationary or periodic flow when there is loss of stability of the laminar flow of a viscous incompressible fluid, J. Appl. Math. Mech. 29 (1965) 